\title{
Fiber-junction design for directional bending sensors
}

\author{
Zhundong $\mathrm{Li}^{1}$, Fengming $\mathrm{Hu}^{1}$, Zhiming Chen $\mathbb{D}^{1,2 凶}$, Jingcheng Huang ${ }^{2}$, Guoning Chen ${ }^{3}$, Runbo Chen ${ }^{1}$, Minmin Wei ${ }^{1}, \mathrm{Kete} \mathrm{Lao}^{1}$, \\ Jiajia Hu${ }^{1}$, Jintao Zheng ${ }^{1}$, Lvfei Wang ${ }^{4}$, Yuan Yao ${ }^{4}$, Xiaoyan $\mathrm{Hu}^{1}$, Baowen Liang ${ }^{1}$, Min Yang ${ }^{3}$, Xihong Lu (D) ${ }^{1}$, Jinxiu Wen ${ }^{1}$ and \\ Jianyi Luo iD ${ }^{1,2 凶}$
}

Flexible sensors in wearable electronics have become increasingly multifunctional due to the development of materials synthesis and structure design. In particular, structural design can not only add capabilities to sensors fabricated from existing available and normal materials, but also offer opportunities for the fabrication of sensors with certain desired functions. Here, we designed a series of fiber-junction structure models, in which two fibers were simply hooked to each other to form a junction on a flexible printed circuit, for fabrication of directional bending sensors. The value and direction of bending angle are related to the change in electronic signal by a theoretical expression, allowing us to employ a simple and practicable method to use available conductive fiber materials to fabricate high-sensitivity, high-resolution and directional bending sensors. In addition, these models are generally applicable, which have broad combination with different conductive fiber, and corresponding bending sensors all possess capability of directional identification. Furthermore, the capability of identifying directional bending was demonstrated by human motion monitoring such as joint bending and muscle contraction.

npj Flexible Electronics (2021)5:4; https://doi.org/10.1038/s41528-021-00102-2

\section{INTRODUCTION}

Recent advances in the development of flexible sensors with multifunction have opened a landscape for wearable electronics, meeting the increasing diversification of human demand ${ }^{1-6}$. Two major strategies have driven the multifunction of flexible sensors ${ }^{7}$. The first strategy is the design and synthesis of materials, including metallic nanoparticles and nanowires ${ }^{8-10}$, liquid metal ${ }^{11}$, conductive polymers ${ }^{12,13}$, and carbon-based micro/nanomaterials $^{14-16}$. Material innovation is a great tool to extend the sensing function of flexible sensors, but the expected industrial cost of materials is not easily estimated. The second strategy is structure design, through which researchers can use existing commercially available materials to fabricate multifunctional flexible sensors ${ }^{17}$. Furthermore, structure design allows for selecting low-cost and suitable materials to fabricate sensors with target functions.

Structure design refers to the construction of various geometric configurations, and these configurations can be divided into two types: in-plane and out-of-plane ${ }^{18}$. For example, in-plane configurations such as serpentine shapes ${ }^{19,20}$, fractal geometries ${ }^{17}$, mesh-like geometries ${ }^{21}$, and buckled structures ${ }^{22}$ can be obtained by thin film deposition and etching. Coiled shapes ${ }^{23,24}, 3 D$ wavy $^{25,26}, 3 \mathrm{D}$ mesostructures ${ }^{27-29}$, and wrinkled structures ${ }^{30-32}$ belong to the out-of-plane configurations. Typical examples of wrinkled structures are the application of conductive fibers in stretch strain or bending sensors ${ }^{30,31}$. The conductive fiber is made from metallic nanoparticles and nanowires, conductive polymers, carbon-based micro/nanomaterials, or conductive material-modified conventional fibers ${ }^{16}$. The inherent characteristics of the softness and flexibility of conductive fiber make them particularly suitable for the fabrication of stretch strain or bending sensors ${ }^{33,34}$. Fiber-based stretch-strain or bending sensors have attracted wide attention for their potential applications in human motion monitoring, human rehabilitation, and human-machine interaction $^{35-39}$. However, sensors based on a single fiber mostly have a sole function, such as mainly focusing on detecting a single bending direction ${ }^{37-39}$. These single directional detections limit the sensors in directional motion monitoring and human-machine interaction with multi-axis robots. To address this issue, one strategy is to convert aligned fibers into a fiber network with wrinkled microstructures to fabricate a sensor with the function of detecting of multidirectional bending ${ }^{40,41}$. However, this strategy often involves a complicated multistep fabrication process. Thus, it is necessary to develop a simple structural model for the fabrication of directional bending sensors for application in future wearable electronics.

Here, we introduce a series of fiber-junction structure models for the fabrication of directional bending sensors. These models can be divided into $\mathrm{X}_{1}$-junctions, $\mathrm{X}_{2}$-junctions, $\mathrm{K}_{1}$-junctions and $\mathrm{K}_{2}$ junctions and $\mathrm{H}$-junctions, which are all formed by simply connecting two commercially available conductive fibers in a hook-like fashion. The bending sensor based on these fiberjunction structures showed high sensitivity, high-resolution, linearity, and long-term stability. Moreover, variations in the resistance of these sensors show the opposite trend for outward or inward bending, illustrating the ability of the sensors to recognize the bending direction. We further demonstrate this capability by successfully applying these sensors in both large-size and subtle bending strain human motion monitoring, indicating great promise in fiber-junction structure-based bending sensors.

\section{RESULTS}

\section{Fiber-junction structure modes and operating principle}

Supplementary Fig. 1 shows a series of fiber-junction structure models, including $\mathrm{X}_{1}$-junction, $\mathrm{X}_{2}$-junction, $\mathrm{K}_{1}$-junction and $\mathrm{K}_{2}$ junction and $\mathrm{H}$-junction with similar layout in their junction structure. The junction formation involved two commercially available conductive fibers. Different junction models constructed

\footnotetext{
${ }^{1}$ Research Center of Flexible Sensing Materials and Devices, School of Applied Physics and Materials, Wuyi University, Jiangmen, China. ${ }^{2}$ WYU-Flexwarm Joint Lab for Flexible Sensing Technologies, Guangdong Flexwarm Advanced Materials \& Technology Co., Ltd, Guangzhou, China. ${ }^{3}$ Faculty of Intelligent Manufacturing, Wuyi University, Jiangmen, China. ${ }^{4}$ School of Art and Design, Wuyi University, Jiangmen, China. ${ }^{凶}$ email: z-m.chen@qq.com; luojiany@mail3.sysu.edu.cn
} 


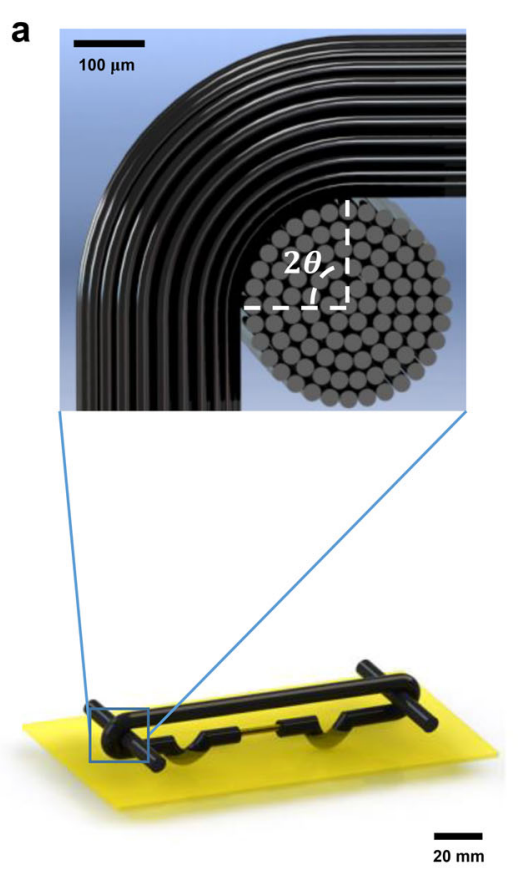

Relax b

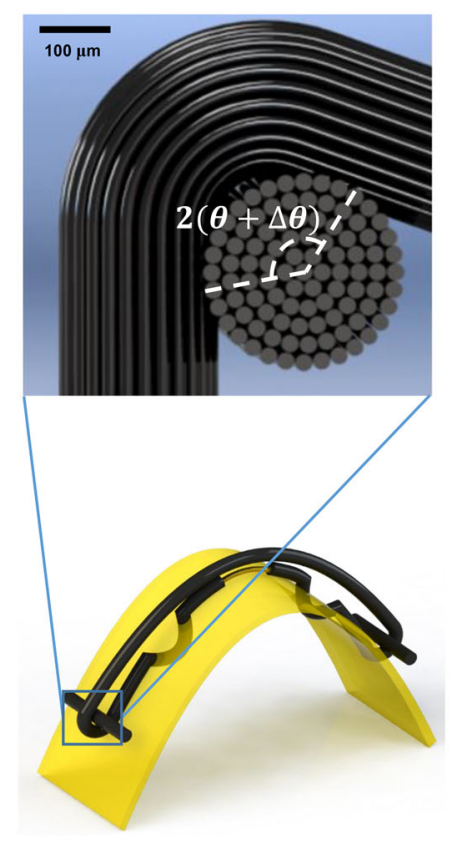

Outward bending

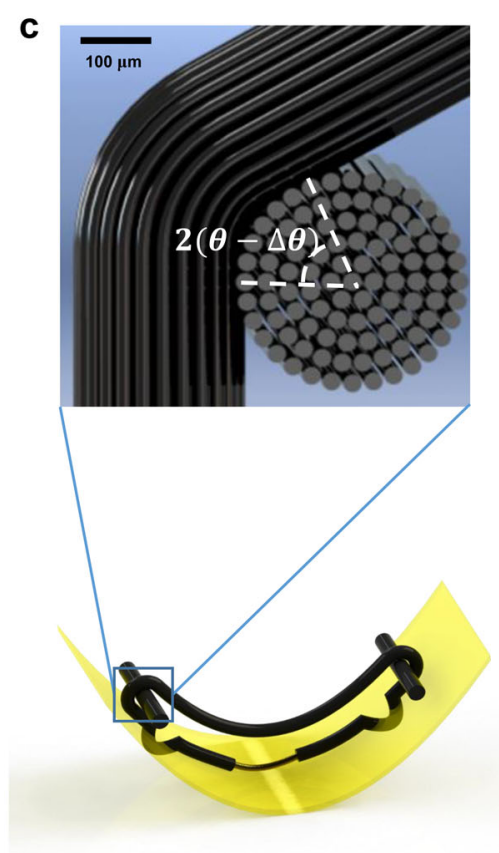

Inward bending

Fig. 1 H-junction structure model for bending sensor. Response in contact area and contact angle of two adjacent fibers of the junction when the bending sensor at a relaxing, $\mathbf{b}$ outward bending, and $\mathbf{c}$ inward bending.

with conductive fibers in a flexible substrate were attributed to different connected configurations of these fibers. Taking the $\mathrm{H}-$ junction structure model as an example, the conductive fibers were fixed on the PI substrate by prearranged copper holes and then formed two junctions on both ends of the substrate. As illustrated in Fig. 1a, once the designed sensor was fabricated, two fibers were brought in contact each other at a contact angle $\theta$, which could be demonstrated by the SEM image of the crosssection of the fiber beams junction. In our designed model, the fabricated sensor possessed double-directional bending modes: outward bending and inward bending. During outward bending, the conductive fiber fixed transversely on the substrate underwent tensile strain, which resulted in stretching of the longitudinal conductive fiber. Thus, the two fibers at the junctions became tighter, resulting in an expansion of the contact area and an increase in the contact angle (Fig. 1b). In contrast, inward bending compressed the transverse fiber, therefore, the two fibers at the junctions became looser with a reduced contact area and contact angle (Fig. 1c). In addition, according to the quantum tunneling effect, the relationship between the resistance change rate of these model-based sensors and the contact angle can be expressed as (for the derivation, see Supplementary Note 1).

$\ln \left(\frac{\Delta R}{R}+1\right)=-K \Delta \theta$.

where the $\frac{\Delta R}{R}$ is the resistance change rate of the sensor, and $K$ is a constant of a certain sensor. This means that $\ln \left(\frac{\Delta R}{R}+1\right)$ is linear with $\Delta \theta$. Under the assumption that the contact angle $\theta$ increases linearly with the bending angle $a$, and $\Delta \theta$ is equal to $a / B, B$ is the angle reduction factor. Thus, we can obtain the relationship between the resistance change rate and the bending angle in the bending sensor as follows:

$\ln \left(\frac{\Delta R}{R}+1\right)=-\frac{K}{B} a$.

The bending angle $a$ is positive as the sensor bends outward, and the angle of inward bending is assumed to be negative.
Because $\ln \left(\frac{\Delta R}{R}+1\right)$ is negatively linearly correlated with the bending angle $a$, the electrical signals of the sensor evolve in a negative half in case of outward bending, while they evolve in the opposite half in case of inward bending. Therefore, our designed structure model allowed the sensor to undergo double-directional bending modes to recognize the directions of human motions. In addition, the $\mathrm{H}$-junction structure can improve the sensitivity of the sensor due to the changes in resistance caused by variations in the contact area and contact angle of the two fibers instead of relying on stretching-like one-dimensional structure fiber-based bending sensors ${ }^{40}$. Similarly, $\mathrm{X}_{1}$-junction, $\mathrm{X}_{2}$-junction $\mathrm{K}_{1}$-junction and $\mathrm{K}_{2}$-junction structure models can also use Eqs. (1) and (2) to explain the relationship between their relevant resistance change rate and the bending angle of the sensor because all these fiberjunction structure models have similar cross junctions. Moreover, a cascade is another strategy to enhance the sensitivity of the bending sensor, and a more significant response in resistance variation can be observed in cascading structure sensors. As shown in Supplementary Fig. 3, the fiber-junction bending sensors can be easily constructed into a large-size flexible substrate through cascade, implying that these fiber-junction sensors are potential candidates for future full body-covering electronic skin.

\section{Fiber-junction bending sensor and performance characterization}

To verify the feasibility of the fiber-junction structure models, fiber-junction structure-based bending sensors with conductive carbon fibers as active materials were first fabricated. These fiberjunction bending sensors are composed of conductive fibers and PI substrates, in which conductive fibers with a diameter of $<0.3 \mathrm{~mm}$ were simply threaded through and fixed on a small-size flexible PI substrate with a length of $1.6 \mathrm{~cm}$ and width of $0.8 \mathrm{~cm}$, allowing it to be compatible with the surface of human skin. To demonstrate the above models and perform the curvature characterization of the fiber-junction bending sensor, a highprecision stage with a step motor was used as bend-driver setup. As shown in Fig. 2a, both ends of the sensor were fixed onto the 
a
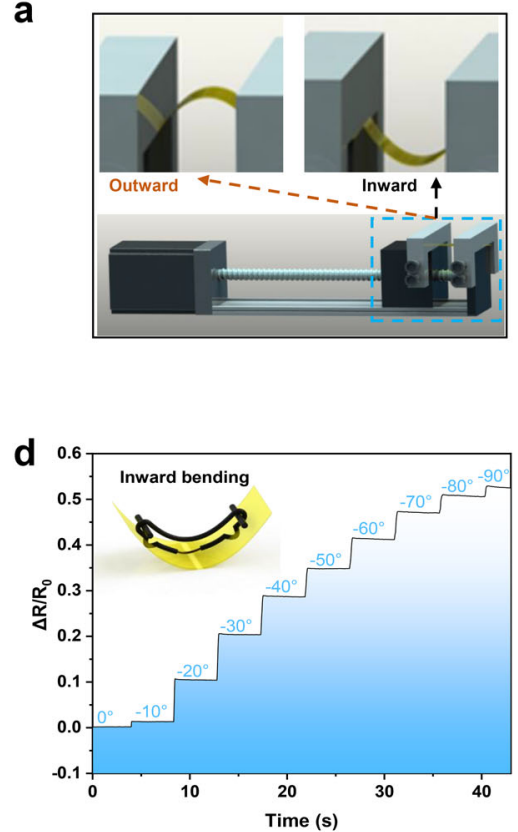
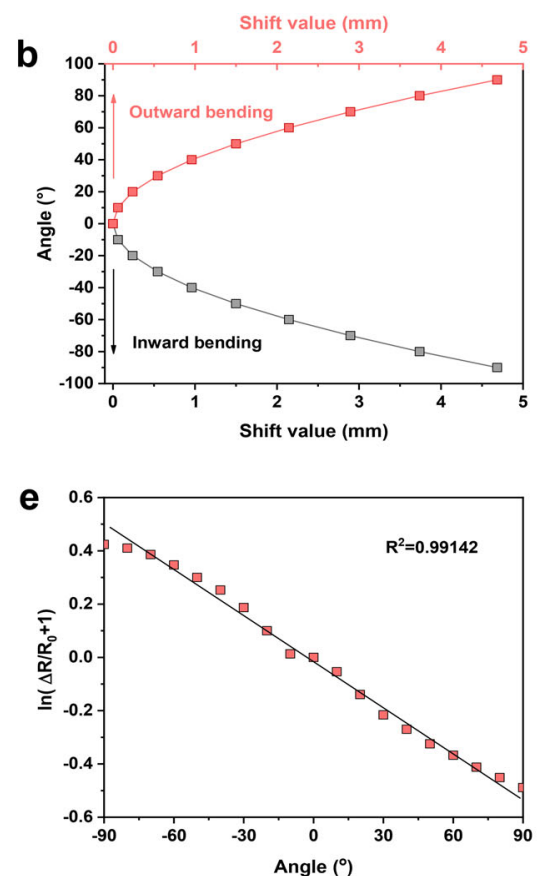

C
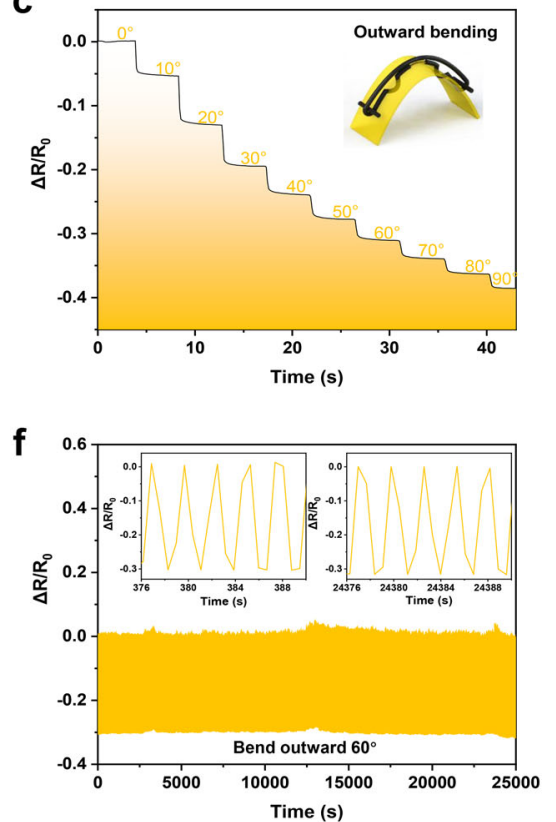

Fig. 2 Performance characterization of bending sensor. a Schematic illustration of the bending sensor fixed to a bend-driver setup in order to detect the response in resistance of the sensor when at outward bending and inward bending. $\mathbf{b}$ Relationship of bending angle and shift value. c Responses in resistance of the $\mathrm{H}$-junction bending sensor when at outward bending. $\mathbf{d}$ Responses in resistance of the $\mathrm{H}$-junction bending sensor when at inward bending. e Value of $\ln \left(\frac{\Delta R}{R}+1\right)$ under various bending angle. $\mathbf{f}$ Responses in resistance of the $\mathrm{H}$-junction bending sensor under outward bending over 10,000 cycles.

stage, and the sensor started outward or inward bending along with a step by step shift. The relation of the bending angle and shift value is shown in Fig. $2 \mathrm{~b}$ and can be expressed by (for the derivation, see Supplementary Note 2)

$\Delta L=L_{0}\left[1-\cos \frac{\alpha}{2}\right]$.

where $L_{0}$ and $\Delta L$ are the initial length of the fiber-junction bending sensor and its shift value, respectively, and $a$ is the bending angle of the sensor, which can be estimated based on the shift value (see Supplementary Fig. 4). As mentioned above, since the initial length of the bending sensor was $1.6 \mathrm{~cm}$, the minimum shift value of each step can be set to $5 \mu \mathrm{m}$. Therefore, the bending angle can be precisely controlled, allowing accurate curvature characterization of the fiber-junction bending sensor.

In the case of the $\mathrm{H}$-junction bending sensor, when driven to outward bending, the resistance change rate of the sensor decreased with increasing bending angle from $0^{\circ}$ to $90^{\circ}$ in $10^{\circ}$ increments (Fig. 2c), suggesting that the outward bending mode led to a decrease in the gaps between the carbon fibers. In addition, this result also indicated that the $\mathrm{H}$-junction bending sensor had the ability to distinguish the bending states of the measured object. In particular, the $\mathrm{H}$-junction bending sensor exhibited a high sensitivity within a small bending strain range that the resistance change rate decreased by $\sim 50 \%$ of the total value of $\Delta R / R_{0}$ as the sensor bent outward to $30^{\circ}$, making it suitable for detecting the small-scale strain of human motions. Moreover, the $\mathrm{H}$-junction bending sensor also show a highresolution under small bending strain that the measure angle can accurate to $1^{\circ}$ when bent outward from $10^{\circ}$ to $19^{\circ}$ (as shown in Supplementary Fig. 5). In contrast to the outward bending, when the sensor bent inward from $0^{\circ}$ to $-90^{\circ}$ (Fig. 2d), the value of $\Delta R /$ $R_{0}$ increase due to the increase in gaps, and the sensor also showed high sensitivity to the initial $30^{\circ}$ inward bending strain. In addition, the other fiber-junction bending sensors, including the $\mathrm{X}_{1}$-junction bending sensor, $\mathrm{X}_{2}$-junction bending sensor, $\mathrm{K}_{1}$ - junction bending sensor and $\mathrm{K}_{2}$-junction bending sensor, all showed similar resistance variations at different bending directions, the resistance change rates were negative when these sensors bent outward, and positive as these sensors bent inward (as shown in Supplementary Fig. 6). These experimental results were consistent with our designed bending sensor model, indicating that this $\mathrm{H}$-junction bending sensor possesses the ability to recognize double-directional bending. Furthermore, the bending directions of the $\mathrm{H}$-junction bending sensor could be determined from its electrical signals. In addition, the $\mathrm{H}$-junction bending sensor fabricated by other commercially available materials, such as graphene fiber and stainless-steel fiber also possessed the capability of identifying double-directional bending (Supplementary Fig. 7), implying the broad combability of our fiber-junction structure models with different conductive fibers. The capability of the bending sensor in detecting directional bending offers a high-precision sensing platform for monitoring of bending strain-based human motions. In addition, according to the relationship of Eq. (2), the bending angles $a$ and their corresponding values of $\Delta R / R_{0}$ from Fig. $2 \mathrm{c}$, d were extracted, and then evaluated by linear fitting. As illustrated in Fig. 2e, there is a well-pronounced linear relationship between $\ln \left(\frac{\Delta R}{R}+1\right)$ and the bending angle $a$. This linear relationship is an intuitive reflection of the accurate bending strain, and facilitates the calibration of the curvature characterization of the $\mathrm{H}$-junction bending sensor. Moreover, this linear relationship further proved that our designed $\mathrm{H}$-junction bending sensor model was reasonable and feasible. In addition, the reproducibility of the linearity of the $\mathrm{H}$-junction bending sensor were evaluated. Three more batches of bending sensors were fabricated, and the linear fitting curves of these sensors were shown in Supplementary Fig. 9. It was found that the $\mathrm{H}$-junction bending sensors with different batches could enable similar stronger linear relationship. In addition to sensitivity and linearity, the linear hysteresis, response time and dynamic bending durability is another three key parameters for the physical robustness and sensing reliability of the bending sensor. 

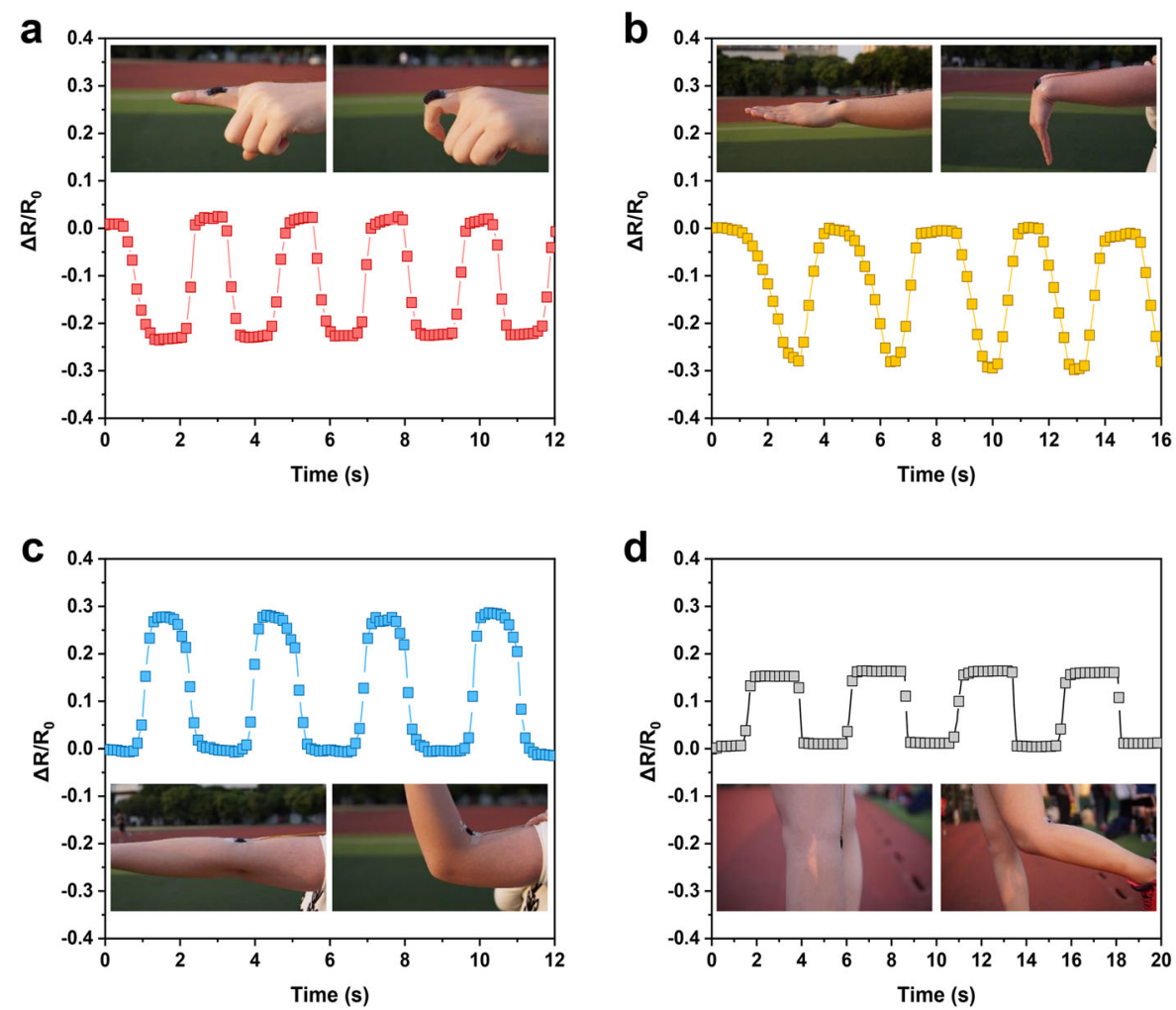

Fig. 3 H-junction bending sensor attached to joints for large-size human motion monitoring. Responses in resistance during the downward bending of (a) finger and (b) wrist, respectively. Responses in resistance during the upward bending of (c) elbow and (d) knee, respectively.

As shown in Supplementary Fig. 10, the H-junction bending sensor presented slight hysteretic behavior by comparing the resistance change rate during the bending outward $60^{\circ} /$ relaxing cycles. The response time determines how quickly the bending sensor responds to the bending strain, and it is usually defined as a $90 \%$ time constant ( $790 \%$ ). For obtaining the response time of the $\mathrm{H}$-junction bending sensor, an equipment that can make the bending sensor bend outward/inward quickly in cycles was fabricated (as shown in Supplementary Note 3 ). The response time of the bending sensor when bent inward from $5^{\circ}$ to $-5^{\circ}$ with a frequency of $9 \mathrm{~Hz}$ was $<30 \mathrm{~ms}$ according to the higher magnification $\Delta R / R_{0}-t$ response curves (Supplementary Fig. 12), and the response time was about $50 \mathrm{~ms}$ for the bending sensor when bent outward from $-5^{\circ}$ to $5^{\circ}$." This fast response ensure the bending sensor apply in the real-time monitoring of fast and complicate signals in the human body. The dynamic bending durability of the $\mathrm{H}$-junction bending sensor was also tested. The real-time evolution of repeated outward bending $60^{\circ}$ and relaxing cycles is shown in Fig. $2 f$. The resistance change rate could still return to the initial value after 10,000 cycles, indicating the longterm structural stability and sensing reliability of the $\mathrm{H}$-junction bending sensor during bending/relaxing cycles. All these results demonstrated that the $\mathrm{H}$-junction bending sensor with excellent properties such as high sensitivity, double-directional bending and long-term dynamic bending durability is a robust candidate for wearable electronics.

\section{Direction large-size motion monitoring}

To evaluate the capability of human motion detection, various kinds of bending sensors have been used to detect joint bending, such as finger bending, wrist bending, elbow bending and knee bending, which are easily detected because of their large-size bending $\operatorname{strain}^{30,32,42}$. However, these bending sensors could not distinguish the direction of joint bending because of the variational tendencies in electrical signals caused by joint bending were identical. According to our designed model, the fiberjunction bending sensors have double-directional bending modes: outward bending and inward bending, corresponding to the downward and/or upward of joint bending. To illustrate the application potential of the fiber-junction bending sensor in human motion monitoring, the $\mathrm{H}$-junction bending sensors representative of the fiber-junction bending sensor were directly attached to joints of the human body. Figure 3a-d show the resistance change rate of the bending sensor adhered to the finger, wrist, elbow, and knee joints. On the one hand, the $\mathrm{H}$ junction bending sensor could precisely capture and identify when the joints were bent or straightened, and it could distinguish the bent position of the joints at different bending angles. As shown in Supplementary Movie 1, the resistance gradually decreased in response to an increasing bending angle of the finger. On the other hand, the bending sensor could recognize the direction of joint bending, Fig. 3a, $b$ show that the downward bending of the finger and wrist caused obvious stretched strain in their corresponding epidermis, in which the resistance change rate of their adhered sensors decreased when the finger and wrist began to bend, and all the values of $\Delta \mathrm{R} / R_{0}$ were negative. In contrast, Fig. 3c, d show that the overall electrical signal of the bending sensors adhered to the elbow and knee was in the positive half, and the resistance change rate increased at first as the sensor bent inward, which was a reflection of the contract strain of the epidermis caused by the upward bending of the elbow and knee. In addition, when the finger, wrist and elbow all bent with a same angle of $60^{\circ}$, the electronic signals of their corresponding sensors shown different response, due to the bending angle of the sensor mounted onto finger was about $60^{\circ}$ but which mounted onto the wrist and elbow were about $30^{\circ}$ (as shown in Supplementary Fig. 13). To vividly demonstrate the 

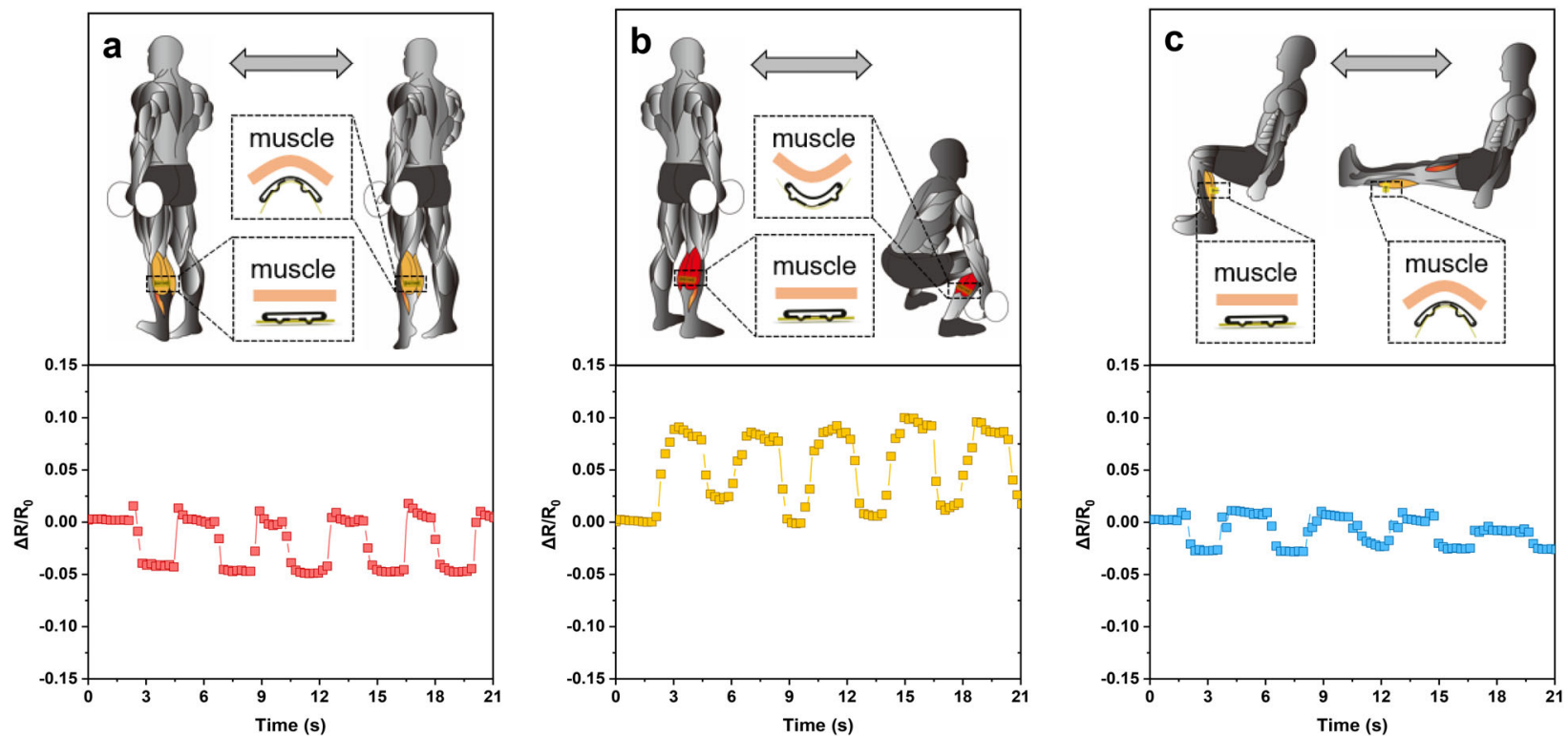

Fig. 4 Subtle motion monitoring by a single channel. $\mathrm{H}$-junction bending sensor reverse horizontally attached to epidermis corresponding to the calf muscle group. Responses in resistance during the fitness exercise of (a) standing heel lifts, (b) squat, and (c) leg extensions, respectively.

ability of the sensor to recognize the direction of joint bending, videos of both downward and upward bending of the wrist were also collected (see Supplementary Movie 2). The bending sensor showed a decreasing resistance and an increasing resistance as the wrist bent downward and upward, respectively, consistent with our designed sensor model. Together, these results provide important insights into the fiber-junction bending sensor possessing the capability of identifying the double-directional bending of human motions.

\section{Direction subtle motion monitoring}

Human motion is based on the combined action of skeletal muscle, bone and joint ${ }^{43}$. Unlike joint bending, which results in a large-size bending strain on the corresponding epidermis, muscle contractions only lead to subtle strain in the corresponding epidermis. However, the epidermis, corresponding to the muscle contraction driven by human motion, is also based on two strain modes: stretching and contracting ${ }^{44}$. The fiber-junction bending sensor with the advantages of exhibiting high sensitivity within a small bending strain range and double-directional bending detection can be mounted on the skin for muscle contraction detection. As illustrated in Fig. $4 \mathrm{a}-\mathrm{C}$, the $\mathrm{H}$-junction bending sensor with a single channel was horizontally attached to the epidermis corresponding to the calf muscles, and three sets of fitness actions, standing heel lifts, squats and leg extensions, were selected to exercise the calf muscles. According to the feedback of the electrical signal of the sensor, the squat led to a more significant deformation of the corresponding epidermis than the other two fitness actions. The resistance change rate was close to 0.1 for the squat, approximately -0.05 for the standing heel lift, and above -0.05 for the leg extension. Thus, the H-junction epiderma sensor was sensitive enough to capture the electrical sign variation when performing muscle motions, and can distinguish optimal fitness actions of a certain muscle. Furthermore, it is noteworthy that the response in the resistance change rate was positive during squatting-standing cycles, while the sensor responded negatively when both heel lifting-relaxing cycles and leg extension-bending cycles were performed. These results can be attributed to the inward bending of the sensor caused by the contract strain of the calf muscles when squatting
(Fig. 4b), and the outward bending due to the stretch strain of the muscles when heel lifts and leg extensions were performed (Fig. $4 a, c)$. This phenomenon can be observed in a real-time video showing a series of fitness actions of standing heel lifts, squats and leg extensions (Supplementary Movie 3), and these experiments confirmed that the $\mathrm{H}$-junction bending sensor had the ability to identify the strain direction of the epidermis for different muscle motions.

In addition to detecting muscle contraction by a single channel, real-time multichannel monitoring of muscle contraction of different muscles was also investigated. Three $\mathrm{H}$-junction bending sensors were attached to the epidermis corresponding to the bicep, tricep, and deltoid muscles and then simultaneously detected the muscle contractions caused by fitness exercises, such as standing dumbbell curls, side lateral raise, push-ups, and shoulder presses (Fig. 5a-d). Standing dumbbell curls induce apparent responses in the resistance change rate of the sensor adhered to the epidermis corresponding to the biceps, which mainly originated from the contract strain of the biceps. However, the response in resistance variation was negligible for the other two sensors which corresponded to triceps and deltoids, indicating that standing dumbbell curl motions might mainly affect the biceps. In contrast to standing dumbbell curls, side lateral raises affect the deltoid muscle, causing a more significant response in the resistance variation. Therefore, the $\mathrm{H}$-junction bending sensor could distinguish which muscles were affected by the fitness exercises, which might be beneficial for providing scientific exercise guidance. In addition, Fig. 5c, d show the response in the resistance variation for each muscle-adhered sensor, implying that both push-ups and shoulder press can act on all three muscles simultaneously. Notably, the evolving trends in the resistance change rate of the sensor adhered to the triceps were opposite to those of the other two sensors (biceps and deltoids). Figure $5 c$ presents the resistance change rate was negative for the sensor corresponding to the triceps, and positive for the others. These results can be attributed to the stretch-strain of the epidermis caused by muscle contraction of the triceps, and the contract strain of both biceps and deltoids under the fitness exercise of push-ups. In contrast to the push-ups, shoulder press lead to opposite evolving trends in the resistance change rate of all the sensor corresponding to the three muscles (see Fig. 5d), 


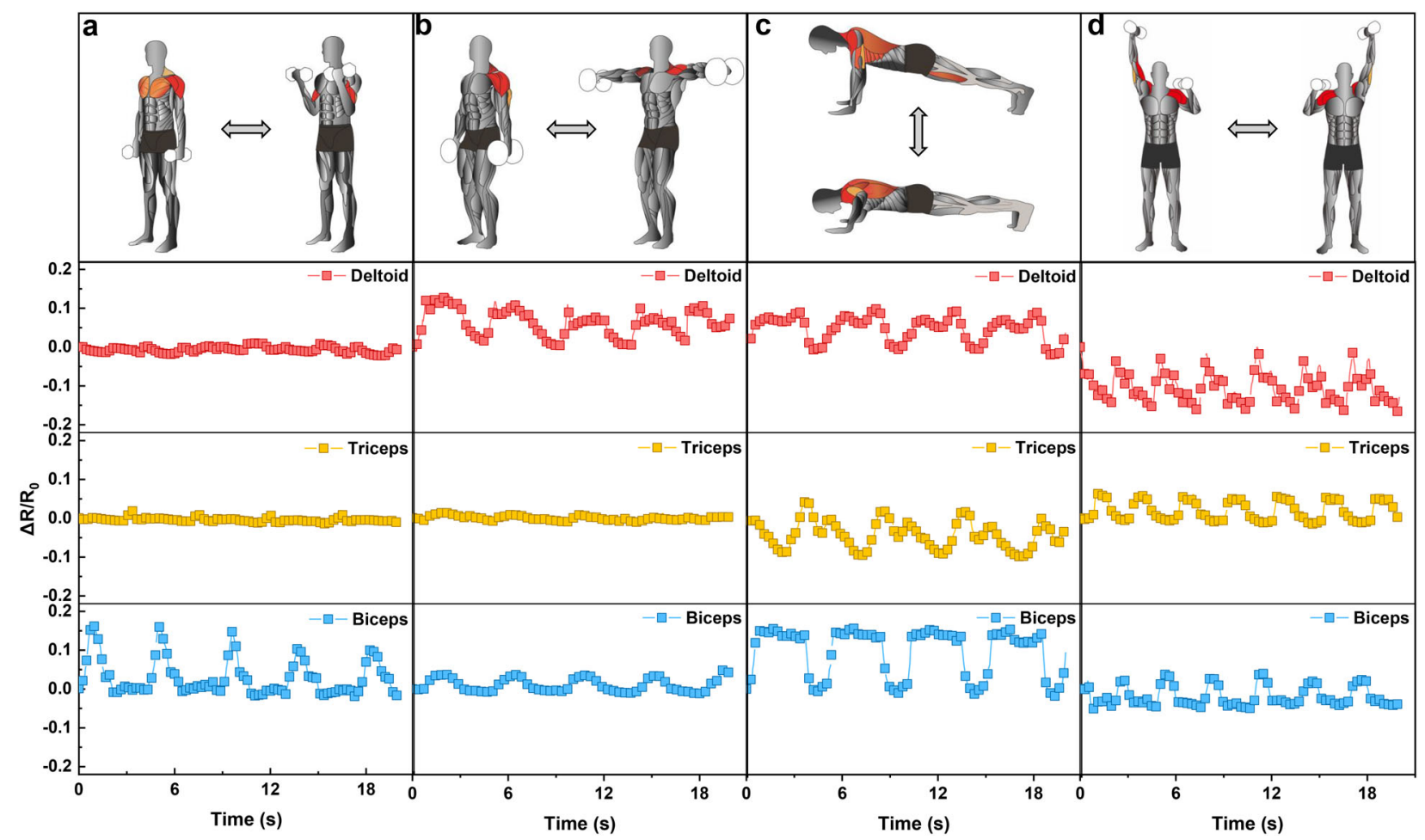

Fig. 5 Subtle motion monitoring by three channels. Three $\mathrm{H}$-junction bending sensors reverse horizontally attached to epidermis corresponding to the biceps, triceps and deltoid muscle at the same time, respectively. Responses in resistance during the fitness exercise of a standing dumbbell curls, $\mathbf{b}$ side lateral raise, c push-ups, and $\mathbf{d}$ shoulder press, respectively.

due to the contract strain of the epidermis caused by muscle contraction the triceps and the stretch-strain of both biceps and deltoids. These experiments further demonstrated that the $\mathrm{H}$ junction bending sensor possesses the capability to identify different strain directions of epidermis movement upon muscle contraction. Notably, these muscle contractions were detected simultaneously by three channels, and the signal crosstalk between the channels was eliminated by circuit design and optimization algorithms (see the signal process, Supplementary Note 3 ). Overall, the combined advantages of high sensitivity and double-directional bending detection indicate that the fiberjunction bending sensor has great potential in wearable electronics for real-time fitness motion monitoring and human-machine interaction.

\section{DISCUSSION}

Based on fiber-junction structure models, a series of bending sensors with excellent sensitivity and double-directional bending modes was designed. The capability of bending direction recognition was derived from the resistance variation in different trends according to the quantum tunneling effect. Other remarkable properties such as high-resolution, linearity, and dynamic bending durability of the obtained $\mathrm{H}$-junction bending sensor were evaluated. The potential of the sensor as wearable electronics in human motion monitoring was also investigated. As expected, the $\mathrm{H}$-junction bending sensor successfully applied to detect large-size bending strains of human motions, such as finger bending, wrist bending, elbow bending, and knee bending. Unexpectedly, the $\mathrm{H}$-junction bending sensor could capture the small movements of muscle contraction and identify the strain direction of the corresponding epidermis. These results indicate that the fiber-junction structure models are practical for sensitive direction bending sensors in wearable electronics, which have potential applications in human motion monitoring, human rehabilitation, and human-machine interaction.

\section{METHODS}

\section{Materials}

Carbon fiber was purchased from Conston Technology Co. LTD; Graphene fiber was purchased from Longsciences Technology Co. LTD; and stainlesssteel fiber was purchased from Shunlong thread Co. LTD.

\section{Fabrication of fiber-junction bending sensor}

The bending sensor is constructed of flexible substrate and conductive fibers, in which a flexible printed circuit board ( $F P C B, P I)$ with a length of $1.6 \mathrm{~cm}$, width of $0.8 \mathrm{~cm}$ as the substrate, and carbon fiber, graphene fiber, and stainless-steel fiber were used as the conductive fiber. For $X_{1}$-junction structure model, $\mathrm{X}_{2}$-junction structure model, $\mathrm{K}_{1}$-junction structure model, and $X_{2}$-junction structure model, two conductive fibers were fixed on the FPCB by four copper holes and formed one junction. Whereas, for $\mathrm{H}-$ junction bending sensor, three fibers were fixed on the FPCB by six copper holes and formed two junctions.

\section{Measurement of the resistance change rate of bending sensor versus bending angle}

A single axis step motor is used for bending sensing test. In the bending calibration experiment, two ends of the bending sensor are fixed on the single axis stepper motor by using the self-made fixture, the program of the stepper motor is set by using the steps corresponding to each angle calculated in advance, and the electric resistance of the sensor is recorded in real time by using a force measuring instrument (Keithley 2400S).

\section{DATA AVAILABILITY}

The experimental data referenced in this text is available from the authors upon reasonable request. 
Received: 12 November 2020; Accepted: 4 February 2021; Published online: 02 March 2021

\section{REFERENCES}

1. Trung, T. Q. \& Lee, N.-E. Flexible and stretchable physical sensor integrated platforms for wearable human-activity monitoring and personal healthcare. Adv. Mater. 28, 4338-4372 (2016).

2. Park, J., Lee, Y., Ha, M., Cho, S. \& Ko, H. Micro/nanostructured surfaces for selfpowered and multifunctional electronic skins. J. Mater. Chem. B 4, 2999-3018 (2016).

3. Son, D. \& Bao, Z. Nanomaterials in skin-inspired electronics: toward soft and robust skin-like electronic nanosystems. ACS Nano 12, 11731-11739 (2018).

4. Xia, Z., Avila, R., Huang, Y. \& Rogers, J. A. Flexible and stretchable antennas for biointegrated electronics. Adv. Mater. 32, 1902767 (2020).

5. Xiang, L. et al. Recent advances in flexible and stretchable sensing systems: from the perspective of system integration. ACS Nano 14, 6449-6469 (2020).

6. Dong, K., Peng, X. \& Wang, Z. L. Fiber/fabric-based piezoelectric and triboelectric nanogenerators for flexible/stretchable and wearable electronics and artificial intelligence. Adv. Mater. 32, 1902549 (2020).

7. Wu, S., Peng, S., Yu, Y. \& Wang, C.-H. Strategies for designing stretchable strain sensors and conductors. Adv. Mater. Technol. 5, 1900908 (2020).

8. Park, l. et al. Nanoscale patterning and electronics on flexible substrate by direct nanoimprinting of metallic nanoparticles. Adv. Mater. 20, 489-496 (2008).

9. Zhao, S. et al. Binary synergistic sensitivity strengthening of bioinspired hierarchial architectures based on fragmentized reduced graphene oxide sponge and silver nanoparticles for strain sensors and beyond. Small 13, 1700944 (2017).

10. Gong, S. et al. Tattoolike polyaniline microparticle-doped gold nanowire patches as highly durable wearable sensors. ACS Appl. Mater. Interfaces 7, 19700-19708 (2015).

11. Dickey, M. D. Stretchable and soft electronics using liquid metals. Adv. Mater. 29, 1606425 (2017)

12. Hwang, B.-U. et al. Transparent stretchable self-powered patchable sensor platform with ultrasensitive recognition of human activities. ACS Nano 9, 8801-8810 (2015).

13. Curry, E. J. et al. Biodegradable nanofiber-based piezoelectric transducer. Proc. Natl Acad. Sci. USA 117, 214-220 (2020)

14. Yamada, T. et al. A stretchable carbon nanotube strain sensor for human-motion detection. Nat. Nanotechnol. 6, 296-301 (2011).

15. Jang, H. et al. Graphene-based flexible and stretchable electronics. Adv. Mater. 28, 4184-4202 (2016).

16. Zeng, W. et al. Fiber-based wearable electronics: a review of materials, fabrication, devices, and applications. Adv. Mater. 26, 5310-5336 (2014).

17. Fan, J. A. et al. Fractal design concepts for stretchable electronics. Nat. Commun. 5, 3266 (2014)

18. Duan, L., D'hooge, D. R. \& Cardon, L. Recent progress on flexible and stretchable piezoresistive strain sensors: from design to application. Prog. Mater. Sci. 114, 100617 (2020).

19. Kim, D.-H. et al. Epidermal electronics. Science 333, 838-843 (2011).

20. Gray, D. S., Tien, J. \& Chen, C. S. High-conductivity elastomeric electronics. Adv. Mater. 16, 393-397 (2004).

21. Guo, F. M., Cui, X., Wang, K. L. \& Wei, J. Q. Stretchable and compressible strain sensors based on carbon nanotube meshes. Nanoscale 8, 19352-19358 (2016).

22. Liu, Z. F. et al. Hierarchically buckled sheath-core fiber for superelastic electronics sensors, and muscles. Science 249, 400-404 (2015).

23. Shang, Y. et al. Super-sttretchable spring-like carbon nanotube ropes. Adv. Mater. 24, 2896-2900 (2012)

24. Son, W. et al. Highly twisted supercoils for superelastic multifunctional fibres. Nat. Commun. 10, 426-436 (2019).

25. Xu, F., Wang, X., Zhu, Y. \& Zhu, Y. Wavy ribbons of carbon nanotubes for stretchable conductors. Adv. Funct. Mater. 22, 1279-1283 (2012).

26. Xu, J., Chen, J., Zhang, M., Hong, J.-D. \& Shi, G. Highly conductive stretchable electrodes prepared by in situ reduction of wavy graphene oxide films coated on elastic tapes. Adv. Electron. Mater. 2, 1600022 (2016).

27. Shyu, T. C. et al. A Kirigami approach to engineering elasticity in nanocomposites through patterned defects. Nat. Mater. 14, 785-789 (2015).

28. Yan, Z. et al. Mechanical assembly of complex, 3D mesostructures from releasable multilayers of advanced materials. Sci. Adv. 2, e1601014 (2016).

29. $\mathrm{Fu}, \mathrm{H}$. et al. Morphable $3 \mathrm{D}$ mesostructures and microelectronic devices multistable buckling mechanics. Nat. Mater. 17, 268-276 (2018)

30. Wei, Y., Chen, S., Yuan, X., Wang, P. \& Liu, L. Multiscale wrinkled microstructures for piezoresistive fibers. Adv. Funct. Mater. 26, 5078-5085 (2016).

31. Sun, $\mathrm{H}$. et al. A highly sensitive and stretchable yarn strain sensor for human motion tracking utilizing a wrinkle-assisted crack structure. ACS Appl. Mater Interfaces 11, 36052-36062 (2019).
32. Xue, P., Chen, C. \& Diao, D. Ultra-sensitive flexible strain sensor based on graphene nanocrystallite carbon film with wrinkle structures. Carbon 147, 227-235 (2019).

33. Yu, J. et al. Adurability study of carbon nanotube fiber based stretchable electronic devices under cyclic deformation. Carbon 94, 352-361 (2015).

34. Zhang, B. et al. Stretchable conductive fibers based on a cracking control strategy for wearable electronics. Adv. Funct. Mater. 18, 1801683 (2018).

35. Fu, Y. et al. Self-powered, stretchable, fiber-based electronic-skin for actively detecting human motion and environmental atmosphere based on a triboelectrification/gas-sensing coupling effct. J. Mater. Chem. C 5, 1231-1239 (2017).

36. Liao, X., Wang, W., Wang, L., Tang, K. \& Zhang, Y. Controllable enhancing stretchability of highly sensitive fiber-based strain sensors for intelligent monitoring. ACS Appl. Mater. Interfaces 11, 2431-2440 (2019).

37. Huang, T. et al. Porous fibers composed of polymer nanoball decorated graphene for wearable and highly sensitive strain sensors. Adv. Funct. Mater. 29, 1903732 (2019).

38. Wang, X., Qiu, Y., Cao, W. \& Hu, P. Highly stretchable and conductive core-sheath chemical vapor deposition graphene fibers and their applications in safe strain sensors. Chem. Mater. 27, 6969-6975 (2015)

39. Wang, R. et al. A bi-sheath fiber sensor for giant tensile and torsional displacements. Adv. Funct. Mater. 27, 1702134 (2017).

40. Chen, S., Song, Y., Ding, D., Ling, Z. \& Xu, F. Flexible and anisotropic strain sensor based on carbonized crepe paper with aligned cellulose fibers. Adv. Funct. Mater. 28, 1802547 (2018)

41. Lee, J.-H. et al. Highly aligned, anisotropic carbon nanofiber films for multidirectional strain sensors with exceptional selectivity. Adv. Funct. Mater. 29, 1901623 (2019)

42. Choi, D. Y. et al. Highly stretchable, hysteresis-free ionic liquid-based strain sensor for precise human motion monitoring. ACS Appl. Mater. Interfaces 9, 1770-1780 (2017).

43. Taga, G. A model of the neuro-musculo-skeletal system for human locomotion. I. Emergence of basic gait. Biol. Cybern. 73, 97-111 (1995).

44. Xu, B. et al. An epidermal stimulation and sensing platform for sensorimotor prosthetic control, management of lower back exertion, and electrical muscle activation. Adv. Mater. 28, 4462-4471 (2016).

\section{ACKNOWLEDGEMENTS}

This work was financially supported by Innovation and Strong School Engineering Fund of Guangdong Province (2017KTSCX186, 2020KQNCX91, and 2020ZDZX2022), Science and Technology Projects of Jiangmen ((2017) 307, (2017) 149, and (2018) 352), Key Laboratory of Optoelectronic materials and Applications in Guangdong Higher Education (2017KSYS011), Science Foundation for Young Teachers of Wuyi University (No. 2018td04), Guangdong Basis and Applied Fundamental Research Fund (2019A1515111190), National Natural Science Foundation of China (12004285), and Hong Kong and Macau Joint Research and Development Fund of Wuyi University (2019WGALH17).

\section{AUTHOR CONTRIBUTIONS}

Z.L., Z.C., J.L. designed research; Z.L., F.H., Z.C., G.C., M.W., K.L., R.C., J.H., J.Z., Y.Y., J.L. performed research; Z.L., F.H., Z.C., J.H., L.W. X.H., B.L., M.Y., X.L., J.W., J.L. analyzed data; and Z.L., Z.C., J.L. wrote the paper.

\section{COMPETING INTERESTS}

The authors declare no competing interests.

\section{ADDITIONAL INFORMATION}

Supplementary information The online version contains supplementary material available at https://doi.org/10.1038/s41528-021-00102-2.

Correspondence and requests for materials should be addressed to Z.C. or J.L.

Reprints and permission information is available at http://www.nature.com/ reprints

Publisher's note Springer Nature remains neutral with regard to jurisdictional claims in published maps and institutional affiliations. 
Open Access This article is licensed under a Creative Commons Attribution 4.0 International License, which permits use, sharing, adaptation, distribution and reproduction in any medium or format, as long as you give appropriate credit to the original author(s) and the source, provide a link to the Creative Commons license, and indicate if changes were made. The images or other third party material in this article are included in the article's Creative Commons license, unless indicated otherwise in a credit line to the material. If material is not included in the article's Creative Commons license and your intended use is not permitted by statutory regulation or exceeds the permitted use, you will need to obtain permission directly from the copyright holder. To view a copy of this license, visit http://creativecommons. org/licenses/by/4.0/.

(c) The Author(s) 2021 